\title{
Hand hygiene adherence according to World Health Organization Recommendations in a Neonatal Intensive Care Unit
}

\author{
Daiane Santos Silva 1 \\ Ana Amelia G. Dourado 2 \\ Caroline Ramos Eustaquio Cerqueira 3 \\ Fernando Hernandez Romero 4 \\ Nair Almeida Amaral 5 \\ Patricia F. Pearce 6 \\ Lívia Pinheiro Pereira 7 \\ Juan Ignácio Calcagno 8
}

1-5,8 Maternidade de Referência Professor José Maria de Magalhães Netto. Salvador, Brasil.
6 Loyola University School of Nursing, New Orleans, LA, United States of America.
7 Escola de Enfermagem. Universidade Federal da Bahia. Rua Dr. Augusto Viana, s/n. Salvador, BA, Brasil. CEP: 40.110-060. E-mail: Iiv_pp@hotmail.com

\section{Abstract}

Objectives: to evaluate the application of hand hygiene technique, according to the World Health Organization (WHO) recommendations, in the neonatal intensive care unit, at a Maternity in Salvador de Bahia, Brazil.

Methods: cross-sectional study. Hand hygiene technique by professional category and alcohol solution consumption were systematically registered. For this task an adapted instrument created by the WHO was used and applied using factsheets. The sample was taken from medical physicians, physiotherapists, nurses and nursing technicians.

Results: hand hygiene adherence regarding WHO recommendations was deficient in terms of technique and in terms of frequency (adequate technique ranged from $0 \%$ to $13.3 \%$ between professional categories). Hand hygiene was frequently ignored (27\% between physicians and $51.8 \%$ between nursing technicians). The moment right after touching surfaces next to patients was the most ignored one. Alcohol gel solution monthly use was only $35 \%$ of the expected value for the unit.

Conclusions: despite the international investigations and efforts for better results, the adherence and compliance to the hand hygiene guidelines is still deficient and continues to be a major problem.

Key words Hand hygiene, Intensive care neonatal, World Health Organization 


\section{Introduction}

For more than 150 years hand hygiene has been researched in the hospital environment as a way of preventing transmission of bacteria, infection and death. ${ }^{1}$ Semmelweis 2 had already demonstrated empirically the reduction of maternal mortality in a maternity ward in Vienna following the use of a chloride solution for hand hygiene.Despite the importance of hand hygiene practice being recognized by health professionals, the low adherence continues being evidenced in the several scientific literatures. ${ }^{3}$

Clinical studies demonstrated the importance of hand hygiene as an effective way of avoiding unnecessary harm to patients by reducing the transmission of pathogens dangerous to health. 4 Adequate hand hygiene is a scientifically proven, easily applied, preventative measure. In this way, the importance of adherence to hand washing protocols, as one of the most important measures for preventing transmission of pathogens in health-care facilities.5-6

Health-care associated infections (HAI) are a great threat for any patient. Low level of adherence to hand hygiene recommendations and protocols continues being a great problem because of the associated risk for pathogen transmission and infection.78 A prevalence rate between $3.5 \%$ and $12 \%$ of HAI in developed countries is estimated by the WHO. ${ }^{9}$ In developing countries, data are scarce and, when present, the levels are generally higher (a prevalence rate of $20.1 \%$ of HAI is estimated). HAI are specially a problem in intensive care units (ICUs) where heavy workload, low compliance with infection prevention and control measures, host reduced defense mechanisms, increased length of stay and invasive procedures such as mechanical ventilation and central venous catheterization are frequent. 10,11

The study was performed in a regional Maternity Hospital located in Salvador, Bahia, Northeastern Brazil. The maternity provides services to high risk pregnant women from all Bahia State and the incidence of HAI at the NICU was of 25.85 for 1000 patients in 2014 and the main microorganisms isolated in blood cultures during the period were Staphylococcus epidermidis (57\%) and Candida parapsilosis $(22 \%)$.

With the objective of improving and enabling adequate adherence to hand hygiene techniques, various international guides have been developed, pointing out moments when hand hygiene is necessary and when alcohol-based hand rub is preferred, the so-called "five moments for hand hygiene".12-13 Despite these international efforts and initiatives, adherence to hand hygiene remains below $50 \%$ in developed countries and continues to be very low amongst physicians and nurses. ${ }^{14-15}$

The Hospital Infection Control Service (HICS) team at the hospital maternity routinely performs hand hygiene trainings and measurement of alcoholbased hand rub solution use at the NICU, according with WHO recommendations. The amount of alcohol-based hand rub solution is used on a worldwide basis as a proxy for measurement of hand hygiene and there is unpublished evidence that the institutional use of alcohol gel in the NICU, is currently below the ideal established by the WHO. The World Health Organization recommends that the minimum expected consumption of alcohol preparation be $20 \mathrm{~mL}$ per patient per day. 16

According to the directives emitted by the Brazilian Ministry for Health and the National Patient Safety Policies, because of high rates of HAI at the NICU and low indicators of alcohol-based hand rub solution use, evaluation of hand hygiene in the NICU is critical to assure appropriate hand hygiene practices are used, and HAI rates reduced.

The purpose of this cross-sectional study was to evaluate hand hygiene practices amongst the professionals of the NICU. The study evaluated the following topics:

1. Hand hygiene technique and adherence to WHO recommendations;

2. Average consumption of alcohol gel solution.

\section{Methods}

The Maternal Hospital is the biggest from Bahia State, in Brazil, and the only care choice for highrisk pregnant women. It has 249 beds and provides specialized services in gyneco-obstetrics, neonatology, pediatric surgery, intensive care medicine and internal medicine. The study was performed at the NICU with a capacity of 20 beds. However, during periods of overcrowding, it could be extended to 24. Care is given to clinical and surgical patients, except neurosurgery. In the unit there is always visibly clean water available, with taps turned on via hands-free mechanism (activated with the elbow), with a total number of five sinks. In all the sinks there was liquid soap ( 5 dispensers) and paper towel was always available. There was also alcohol solution gel available in multiple locations: a) alcoholbased hand rub solution pump dispenser in a tray, located in each one of the beds of the unit and b) 5 wall dispensers. Aside of each sink, there was a poster illustrating adequate soap and water hand hygiene technique. 
An evaluation of techniques for hand sanitization was undertaken during work routines using the "five moments for hand hygiene", variables established by the WHO. The evaluation was undertaken by three trained members of the HICS team in a random manner. For this task an adapted instrument created by the WHO was used and applied using factsheets. 17-18 Data were collected in a systematic manner and date, sex, their professional role, the theoretically necessary hand hygiene moment for the task and the one performed by the staff member during the period of observation registered, according to WHO recommendations.

The sample was taken from medical physicians, physiotherapists, nurses and nursing technicians who worked on weekdays, weekends, and during the three shifts (morning, noon and night). They were selected because they provide routine care for patients. For the sample size it was considered a $90 \%$ confidence and a 0.05 estimate error (271 moments for had hygiene needed). The health-care professionals were individually observed in their daily tasks for periods of 15 minutes during three months after ethics committee approval. To diminish the risk of change in behavior by the staff during the evaluation, they were only told that the HICS would be evaluating the daily routines of the sector, omitting that hand hygiene was the focus of the evaluation.

The five moments for hand hygiene were classified under five categories: a) Not done: the employee under evaluation did not perform any of the five moments needed for hand hygiene according to WHO recommendations; b) Adequate AS: the professional performed adequate technique (duration of the entire procedure and technique employed) for hand hygiene, with alcohol solution, in any of the five moments according to WHO recommendations; c) Inadequate $A S$ : the professional performed inadequate technique for hand hygiene with alcohol solution in any of the five moments; d) Adequate $S \& W$ : the professional performed adequate technique for hand hygiene (duration of the entire procedure and technique employed) with soap and water in any of the five moments according to WHO recommendations; e) Inadequate $S \& W$ : the professional performed inadequate technique for hand hygiene with soap and water in any of the five moments.

The following indicators were calculated: 1) Hand hygiene adherence with alcohol-based hand rub solution or soap and water in the NICU: calculated dividing the number of times that the health professional sanitized their hands by the number of times that they should have done, multiplied by 100; 2) Hand Hygiene with alcohol-based hand rub solu- tion or soap and water in the NICU taking into account the five moments for hand hygiene as specified by the WHO: calculated dividing the number of times in which the health professional sanitized their hand by the number of times that they should have done it multiplied by 100 . This indicator was considered in terms of the five moments for hand hygiene as defined by the WHO; 3) Use of alcohol preparation for hands in the NICU: monitoring the volume of alcohol preparation for hands used for each 1 , 000 patients daily.

The HICS also undertook the monthly calculation of the minimal use of Alcohol-based hand rub solution, in milliliters, in the NICU per month, according to WHO criteria. The value $(46.640 \mathrm{~mL})$ was used as a control parameter for the monthly use of alcohol solution recorded in the NICU.

Categorical variables were reported as proportions (\%). For analysis, the "five moments for hand hygiene" were divided into three groups: a) "not done' for individuals who did not performed hand hygiene; b) "Adequate AS/ S\&W" for individuals who performed adequate hand hygiene with soap and water or alcohol-based hand rub solution; and c) "Inadequate AS/ S\&W" for individuals who performed inadequate hand hygiene.

The research respected the recommendations for privacy and confidentiality by the Resolution ${ }^{\circ} 466$, of the 12th of October, 2012, National Council of Health for Scientific Research with Human Beings and was approved by the Santa Izabel Ethics Board Committee with number CAAE: 51932215.8.0000.5520.

\section{Results}

The evaluated professionals of the Unit were: 9 physiotherapists, 10 physicians, 14 nurses and 20 nursing technicians allocated in 3 shifts (morning, noon, and night) and 274 moments for hand hygiene were registered. Adequate hand hygiene technique whether performed with alcohol-based hand rub or with water and soap, regardless of the moment for hand hygiene, ranged from $0 \%$ between nursing technicians to $13.3 \%$ between physiotherapists. Regardless of professional category and of the moment for hand hygiene that was being evaluated, hand hygiene was frequently ignored $(27 \%$ between physicians and $51.8 \%$ between nursing technicians).

The moment right after touching surfaces proximate to patients was the most ignored moment, regardless of professional category (for every 100 times to be performed was performed only in 33.7). There was no occasion in which nursing technician 
professionals were identified as sanitizing their hands with liquid soap, the adequate technique according to WHO recommendations. Liquid soap and water were preferred for hand hygiene when compared to the use of alcohol-based hand rub solution independent of professional category $(97 \%$ of physicians, $85.7 \%$ of physiotherapists, $92.2 \%$ of nurses and $91.5 \%$ of nursing technicians preferred liquid soap and water). The use of alcohol gel solution for the sanitization of hands was very low and the technique was not practiced adequately by any of the evaluated professionals (Table 1).

\section{Evaluation of Indicators:}

1. Hand hygiene adherence with alcohol gel solution or soap and water: With this indicator the opportunities for hand hygiene (of the total opportunities identified for hand hygiene, the degree to which it was undertaken) were evaluated according to the acting professional. It was identified, for example, that for every hundred times in which hand hygiene was advised, physicians would comply approximately
$70 \%$ ( 23 hand hygiene performed out of 33 opportunities). The category with the least adherence to hand hygiene were the nursing technicians with only $48.1 \%(64 / 133)$ of the opportunities used, be it with the alcohol solution or soap and water, using or not the adequate technique (Figure 1).

2. Hand Hygiene solution of alcohol gel or soap and water in the NICU, taking into account the five moments for hand hygiene as specified by the WHO: There were hand hygiene moments that were more frequently undertaken than others. The moment which presented the greatest compliance was the "sanitization of hands before undertaking clean or aseptic procedures" with $81.2 \%(13 / 16)$. The moment in which health professionals had complied the least, regardless of the professional category evaluated, was "hand hygiene after touching surfaces near to the patient" which was performed 27 times out of 80 opportunities for hand hygiene regardless of professional category or technique used (Table 1).

Figure 1

Indicator of hand hygiene adherence by professional category in the Neonatal Intensive Care Unit.

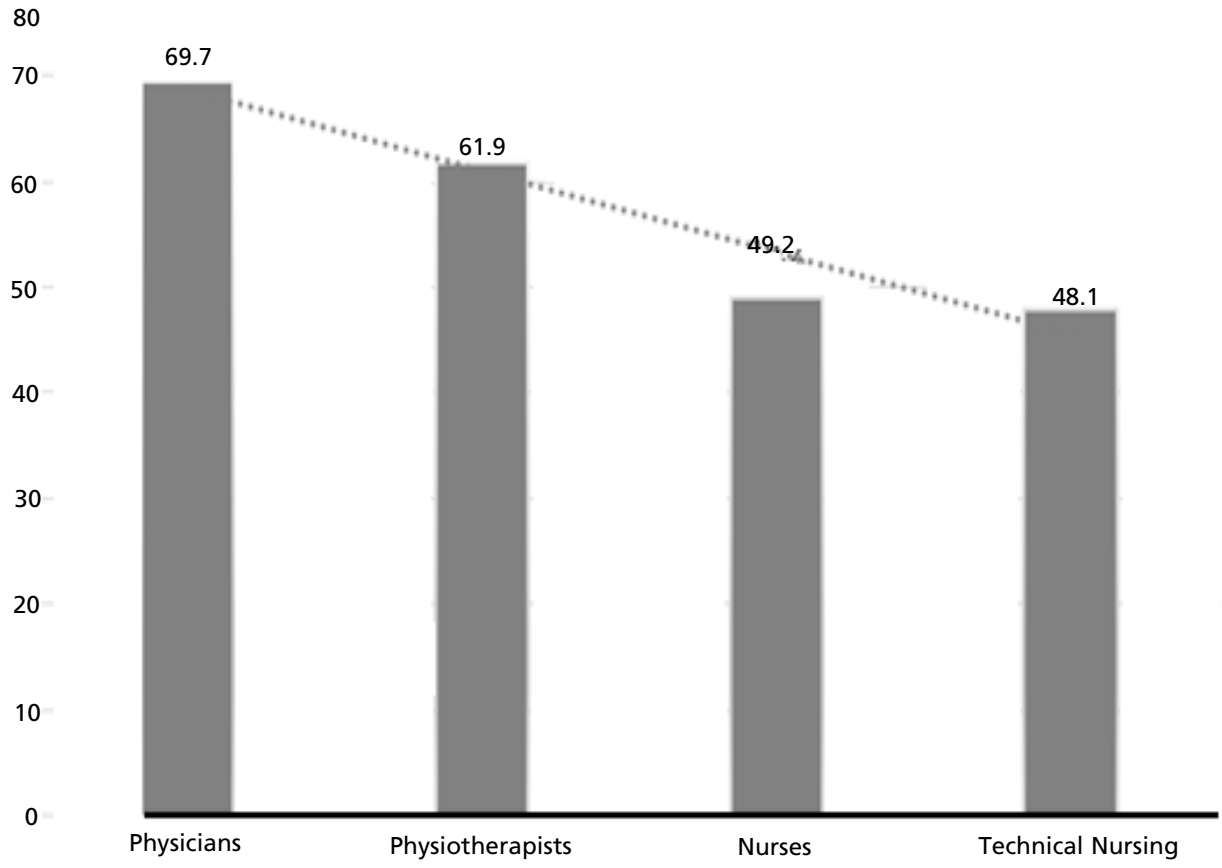




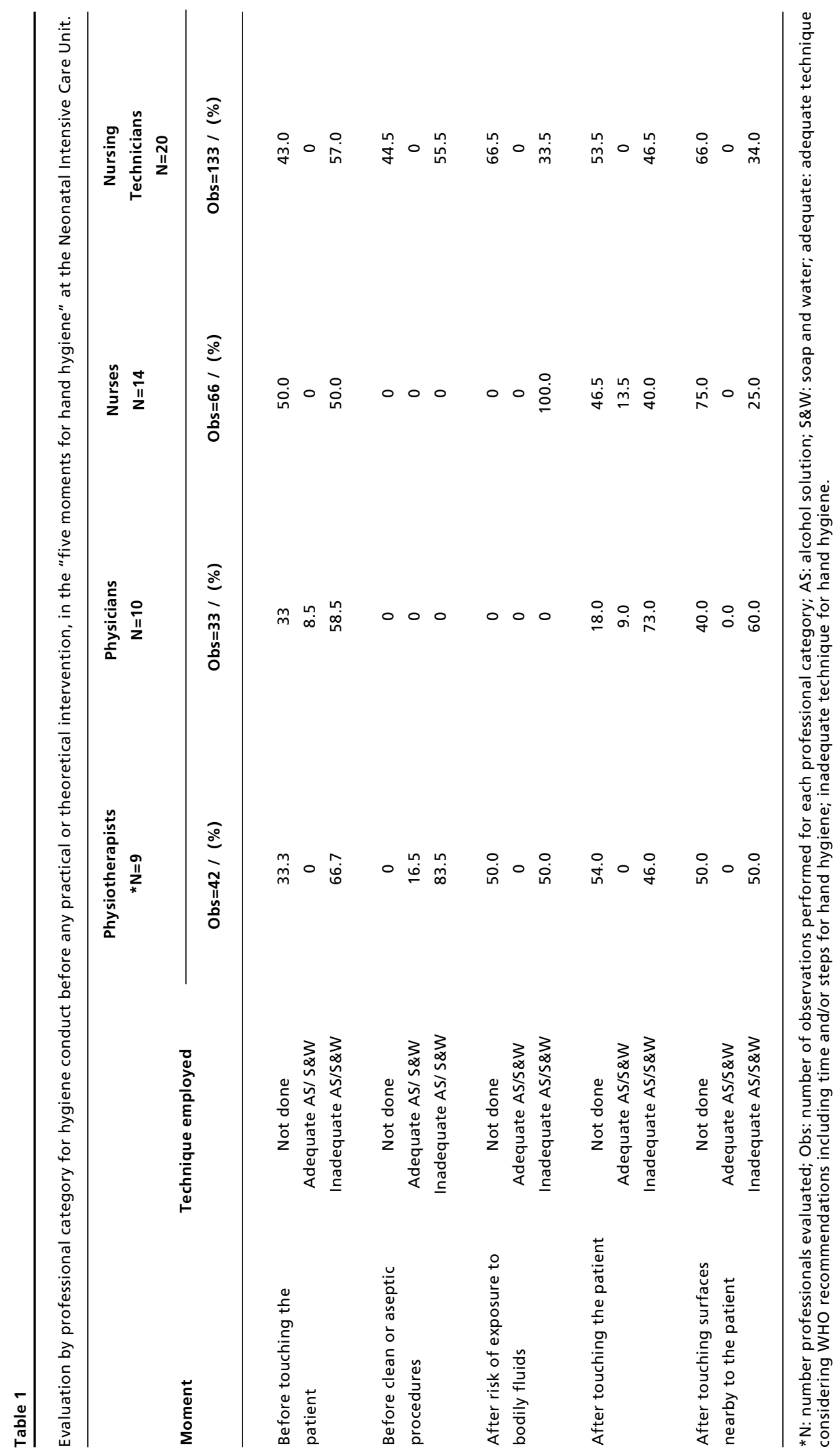


Indicator of hand hygiene adherence taking into account the "five moments for hand hygiene", in the Neonatal Intensive Care Unit .

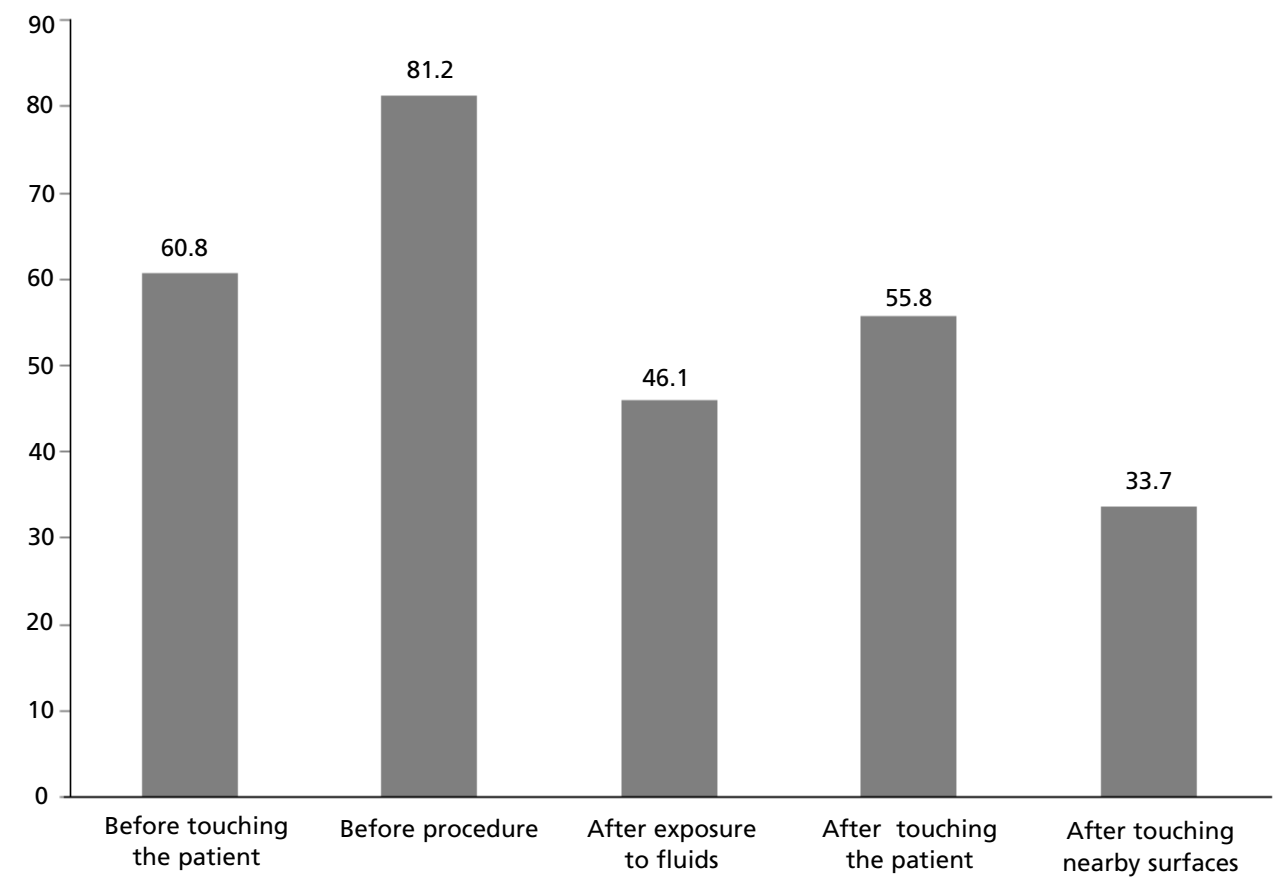

3. Use of alcohol preparation for hands in the Neonatal Intensive Care Unit: The records for the use of alcohol gel solution for hand hygiene in the unit showed a monthly mean of $16.341 \mathrm{~mL}$, much lower than the expected minimum of $46.640 \mathrm{~mL}$, as established by the team of the HICS.

\section{Discussion}

Some limitations should be noted in this crosssectional study. Firstly, hand hygiene was evaluated by direct observation. Professionals did not know of the focus of the team's evaluation, however, there isa possibility of change in their behavior (Hawthorne effect). Hagel et al. ${ }^{19}$ identified that Hawthorne effect is more pronounced in high-performing units but insignificant in low-performance units. This effect is unlikely in our study, due to the high lack ofadherence on the usage of alcohol-based hand rub solution or soap and water in any adequate manner.

Secondly, the indicator developed by the WHO to evaluate professional adherence to hand hygiene recommendations does not take into account exposure duration, or the exposure time each professional category spends with patients. Determination of influence of duration of patient care activities on hand hygiene compliance should be evaluated. In this study, the professional categories which spend more time with the patients, nurses and nursing technicians, had the worst hand hygiene compliance. Several investigators identified that the increase in workload was a negative impact factor on compliance. ${ }^{20-21}$ Variables such as the weariness caused by work routines (severity of patient cases, NICU overcrowding, number of shifts per week, number of jobs) were not registered. Also in this study, significantly more observations were performed with nursing technicians in comparison to physicians, and physicians had the higher compliance rates. Different results were found by some authors like Duggan which published a study with 2,373 hand hygiene observations on physicians and nurses and an inverse correlation was found between the educational level of professionals and the rate of compliance. ${ }^{22}$ In this study, the number of collected observations when considering "physicians" category was small making difficult to distinguish if the better hand hygiene compliance was real or only a random variation. Furthermore, the years spent on education and for professional training, motivation, work expe- 
rience in years and the type of professional education were not registered.

Thirdly, gender was not possible to be evaluated as a factor associated with hand hygiene technique. Only 16 over 274 evaluations were performed by male professionals. The majority of professionals at the maternity are females (only four males at the NICU). Other characteristics, such as years of experience and age of participants, are potential confounders, but were not considered in this study.

Fourthly, evaluations were performed considering only WHO recommendations for hand hygiene. There is no evidence demonstrating WHO recommendations are the best ones for hand hygiene, but they were considered by the team as an international standard of good practice for hand technique. Other techniques could be effective or even better than WHO recommendations, howeverthey were not considered in this study.

Poor adherence to hand hygiene has been reported in other studies. Primo in Goiás, Brazil, performed a before and after study. Before the intervention, 119 opportunities for hand hygiene were collected and the average compliance was $21 \%$, all using water and soap. 23 Similar behavior was identified in our study where the usage rates of alcoholbased hand rub solution were very low. Similar compliance rates were found in high income countries. In a multicenter study involving 13 hospitals in Ontario, Canada,the mean hand hygiene adherence rate of $31,2 \%$ was demonstrated, and adherence was positively associated with being a nurse, availability of alcohol hand rub dispenser and single rooms. 24 Monsalve et al.,25 in an observational study using a sensor network in a medical intensive care unit with 20 beds, at a large university hospital, observed an adherence rate of $20.85 \%$ which increased to $27.9 \%$ when other healthcare worker was present, suggesting influence of peers. WHO identified results in line with the above findings. Health-care facilities (807) from 91 countries (12 from Brazil) have had completed a Hand Hygiene SelfAssessment Survey (HHSAF). The objective was to assess and track progress in hand hygiene improvement, including indicators in five categories (system change, training and education, evaluation and feedback, reminders in the workplace and institutional safety climate for hand hygiene). Each indicator received a score adding up to a maximum of 100 points for each category (maximum score 500 points). Participating facilities were mostly public (70\%) and hospitals (77\%). The lowest mean score was recorded in the African Region (280.9 \pm 127.3$)$, while the highest was in the South-East Asia Region
(420.6 \pm 77.6$)$, representing 60 and 231 health care facilities, respectively. Among the sections, the lowest scores concerned evaluation and feedback on hand hygiene activities and the institutional patient safety climate. 26

The multi-faceted and complex nature of HAI is such that hand hygiene is necessary, but not sufficient alone to reduce HAI. According to Huynh et al. 27 the incidence of neonatal infection in developing countries is the leading cause of death in children under 5 years due to a number of risk factors like the emergence and spread of antibiotic resistance.

In this study, water, soap, alcohol gel, and paper towel were provided appropriately and no obstructions for the use of these elements were in evidence. Adherence to hand hygiene was deficient as much in terms of technique as in terms of frequency with which this preventative measure for infections should have been carried out, according to the WHO criteria. The use of alcohol gel solution was much less than that indicated following the evaluation of the hospital's HICS.

The hand hygiene technique and adherence to WHO recommendations were deficient in this study. The rationale for not adhering to the recommended guidelines were not searched and were not the objective of this study. Hand hygiene continues to be a low cost, easy access, efficient strategy for the prevention of transmission of bacteria and HAI but the adherence and compliance to the hand hygiene guidelines is still deficient and continues to be a major problem worldwide mainly in low and middle income countries.

Despite the international investigations and efforts for better results and considering the current clinical evidence, it seems necessary that greater efforts should be performed to implement appropriate research and interventions to improve the professional compliance of infection control processes and recommendations for hand hygiene.

Improve hand hygiene adherence and technique following WHO recommendations in an effective practice may reflect better quality care. However, it is still necessary to stimulate awareness and to promote an institutional culture of patient safety,which among other things, improves adhesion and hand hygiene technique. Thus, it is suggested that, in addition to the professional and individual commitment, it is also needed managers and leaders' commitment and alignment of responsibilities in actions such as: constant educational campaigns, mobilizing actions, operational and structural support. 


\section{References}

1. Rotter M. Hand washing and hand disinfection. In: Mayhall $\mathrm{CG}$, editor. Hospital epidemiology and infection control. 2 ed. Philadelphia, PA: Lippincott Williams \& Wilkins ;1999. p. 1339-55.

2. Semmelweis I. Etiology, concept, and prophylaxis of childbed fever (History of Science and Medicine). The University of Wisconsin Press; 1983.

3. Sousa ECP, Silva FL. The knowledge and the accession of practice hand hygiene of health professionals: literature review. Rev Saúde Foco. 2016; 3 (1): 84-93.

4. Allegranzi B, Pittet D. Role of hand hygiene in healthcareassociated infection prevention. J Hosp Infect. 2009; 73 (4): 305-14.

5. Girou E, Legrand P, Soing-Altrach S, Lemire A, Poulain C, Allaire A, Tkoub-Scheirlinck L, Chai SH, Dupeyron C, Loche CM. Association between hand hygiene compliance and methicillin-resistant Staphylococcus aureus prevalence in a French rehabilitation hospital. Infect Control Hosp Epidemiol. 2006. 27 (10): 1128-30.

6- Brasil. Agência Nacional de Vigilância Sanitária. Segurança do Paciente em Serviços de Saúde: Higienização das Mãos / Agência Nacional de Vigilância Sanitária. Brasília: Anvisa; 2009.

7. WHO (World Health Organization). Benedetta Allegranzi, Sepideh Bagheri Nejad, Gabriela Garcia Castillejos, Claire Kilpatrick, Edward Kelley, Elizabeth Mathai. Report on the Burden of Endemic Health Care-Associated Infection Worldwide. Clean care is safer care. 2011

8. Pires D, Soule H, Bellissimo-Rodrigues F, Gayet-Ageron A, Pittet D. Hand Hygiene With Alcohol-Based Hand Rub: How Long Is Long Enough? Infect Control Hosp Epidemiol. 2017; 38(5): 547-552.

9. WHO World Alliance for Patient Safety. Health-Care Associated Infections. Available at: http://www.who.int/ gpsc/country_work/gpsc_ccisc_fact_sheet_en.pdf.

10. Alp E, Damani N. Healthcare-associated infections in intensive care units: epidemiology and infection control in low-to-middle income countries. J Infect Dev Ctries. 2015; 9 (10): 1040-5

11. Fortaleza CMCB, Padoveze MC, Kiffer CRV, Barth AL, Carneiro ICDRS, Giamberardino HIG, Rodrigues JLN, Santos Filho L, de Mello MJG, Pereira MS, Gontijo Filho PP, Rocha M, Servolo de Medeiros EA, Pignatari ACC. Multi-state survey of healthcare-associated infections in acute care hospitals in Brazil. J Hosp Infect. 2017; 96 (2): 139-44.

12. Oliveira AC, Gama CS, Paula AO. Multimodal strategy to improve the adherence to hand hygiene and self-assessment of the institution for the promotion and practice of hand hygiene. J Public Health (Oxf). 2017; 1-6. doi: 10.1093/pubmed/fdx035

13. Farinaz Farhoudi, Anahita Sanaei Dashti, Minoo Hoshangi Davani, Nadiyeh Ghalebi, Golnar Sajadi, Raziyeh
Taghizadeh. "Impact of WHO Hand Hygiene Improvement Program Implementation: A Quasi-Experimental Trial. BioMed Res Int. 2016; Article ID 7026169, 7 pages. doi:10.1155/2016/7026169

14. Wetzker W, Bunte-Schönberger K, Walter J, Pilarski G, Gastmeier P, Reichardt Ch. Compliance with hand hygiene: reference data from the national hand hygiene campaign in Germany. Journal of Hospital Infect. 2016; 92 (4): 328-31.

15. Kingston L, O'Connell NH, Dunne CP. Hand Hygienerelated clinical trials reported since 2010: a systematic review. J Hosp Infect. 2016. 92 (4): 309-320.

16. Brasil. Ministério da Saúde. Anvisa. Fiocruz. Anexo 01: Protocolo para a prática de higiene das mãos em serviços de saúde. Protocolo integrante do Programa Nacional de Segurança do Paciente. 09/07/2013.

17. WHO (World Health Organization). To collect data about structures and resources at ward level; 2009.

18. WHO (World Health Organization). A form to complete when monitoring hand hygiene; 2009.

19. Hagel S, Reischke J, Kesselmeier M, Winning J, Gastmeier P, Brunkhorst FM, Scherag A, Pletz MW. Quantifying the Hawthorne Effect in Hand Hygiene Compliance Through Comparing Direct Observation With Automated Hand Hygiene Monitoring. Infect Control Hosp Epidemiol. 2015; 36 (8): 957-62.

20. Tomar S, Lodha R, Kapil A. Hand hygiene compliance of healthcare workers in a pediatric intensive care unit. Indian Pediatr. 2015; 52 (7): 620-1.

21. Sharma S, Sharma S, Puri S, Whing J. Hand hygiene compliance in the intensive care units of a tertiary care hospital. Indian J Community Med. 2011; 36 (3): 217-21.

22. Duggan JM, Hensley S, Khuder S, Papadimos TJ, Jacobs L. Inverse correlation between level of profesional education and rate of handwashing compliance in a teaching hospital. Infect Control Hosp Epidemiol. 2008; 29 (6): 534-8.

23. Primo MGB, Ribeiro LCM, Figueiredo LFS, Sirico SCA, Souza MA. Adesão à prática de higienização das mãos por profissionais de saúde de um Hospital Universitário. Rev Eletr Enf. 2010; 12 (2): 266-71.

24. Mertz D, Johnstone J, Krueger P, Brazil K, Walter SD, Loeb $\mathrm{M}$. Adherence to hand hygiene and risk factors for poor adherence in 13 Ontario acute care hospitals. Am J Infect Control. 2011; 39 (8): 693-6.

25. Monsalve MN, Pemmaraju SV, Thomas GW, Herman T, Segre AM, Polgreen PM. Do Peer Effects Improve Hand Hygiene Adherence among Healthcare Workers? Infect Control Hosp Epidemiol. 2014; 35 (10): 1277-85.

26. Kilpatrick C, Allegranzi B. Summary Report: Hand Hygiene Self-Assessment Framework Survey 2016. A report from the WHO Infection Prevention and Control Global Unit. Available at: http://www.who.int/ gpsc/5may/hand-hygiene-report.pdf. 
27. Huynh B-T, Padget M, Garin B, Herindrainy P, Kermorvant-Duchemin E, Watier L, Guillemot D,

Delarocque-Astagneau E. Burden of bacterial resistance among neonatal infections in low income countries: how convincing is the epidemiological evidence? BMC Infect Dis. 2015; 15: 127.

Received on January $11^{\text {th }}, 2017$

Final Version Presented on May 17th, 2017

Approved on June 20th, 2017 